\title{
DEVELOPING A TRAINING PROGRAM IN HEALTH IMPACT ASSESSMENT (HIA) IN PORTUGAL
}

Costa, A. ' Costa, L. ' Gulis, G. ${ }^{2}$ Nowacki, J. ${ }^{3}$ Caldas de Almeida, T. 1

' National Institute of Health Doutor Ricardo Jorge (INSA), Lisboa, Portugal; ${ }^{2}$ University of Southern Denmark, Esbjerg, Denmark; ${ }^{3}$ WHO Regional Office for Europe, Bonne, Germany

\section{QVERVIEW:}

Under the scope of the Biennial Collaborative Agreement between WHD and the Portuguese Ministry of Health, the National Health Institute, initiated a capacity building training program.

The new Public Health Law proposal, which is being discussed at the Portuguese Parliament, proposes mandatary HIAs at the national level.

A workshop was held in Lisban in November 2017, including a Policy Dialogue between Health and other sectars, to start the training programme.

\section{OBJETIVES:}

To build technical expertise and capacity to assess and evaluate policies, plans, programs and projects using HIA methadology.

To develop a HIA toolkit, validated for use in Portuguese context based an national case studies.

\section{TRAINING PRDGRAM STRATEgY}

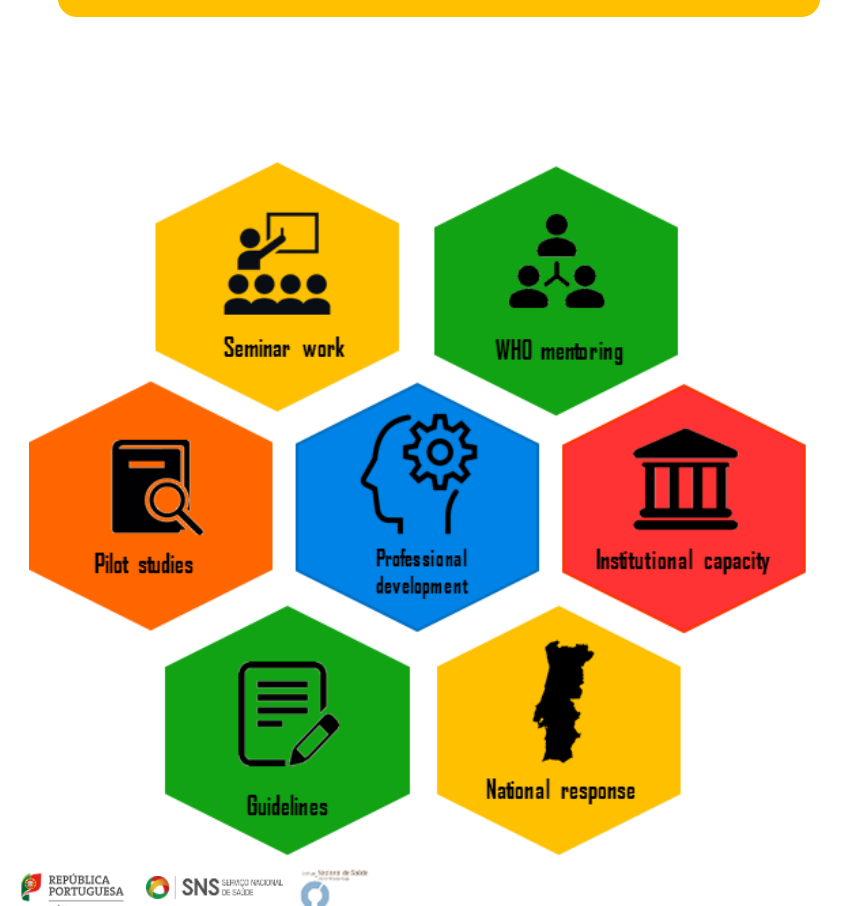

\section{PILDT STUDIES: A LEARN BY DDING PRDCESS}

HIA methadalogy using the guidelines of Irish National Institute of Public Health
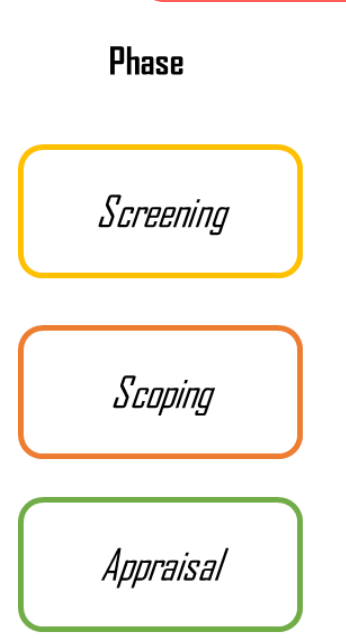

Recomendations
Tasks

Initial investigation:

- potential health impacts of a policy, program or project

- potential impacts on vulnerable sections of the population

Foundation for the rest of the assessment: - selection and constitution of a steering group - develop a work plan and the Terms of Reference

Deeper investigation

- Community profle

- Data collection

- Impact analysis: data collected and assessed

- Priority health impacts identification

Comunication of the findings

- Decision-makers

- Stakeholders

- Population

\section{Main outputs}

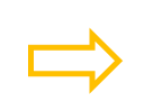

Interim Report

Decision-making Protocol Terms of Reference Work Plan Report

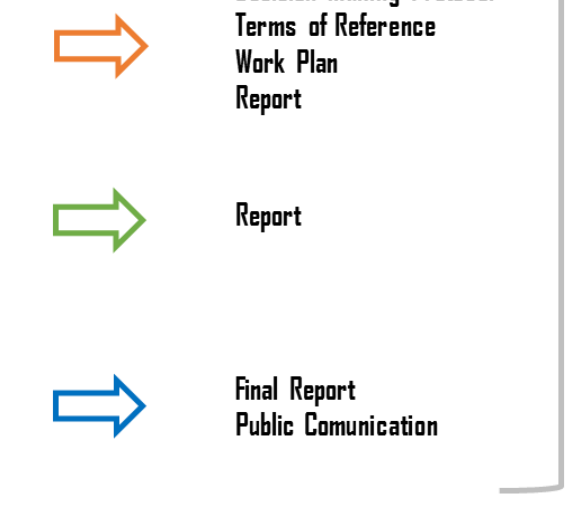

\section{CONCLUSIONS:}

Impact at short term: To obtain well trained professionals able to develop HIA and tonls required and fit to the national, regional and local characteristics.

Impact at medium term: To contribute to the HIA Partuguese madel that is being developed, as an instrument of populationbased health planning.

Impact at lang term: To create a netwark with infarmed stakeholders that became an instrument far tackling HIA in Partugal. 